\title{
Prediction of cesarean hysterectomy in placenta previa complicated with prior cesarean: a retrospective study
}

\author{
Bin Liu ${ }^{1 \dagger}$, Songqing Deng ${ }^{1 \dagger}$, Meifang Lin², Yimin Chen ${ }^{3}$, Jian Cai', Jianbo Yang ${ }^{1}$, Jinxin Zhang ${ }^{3}$, Jianjian Cui ${ }^{1}$,
} Lixia Shen ${ }^{1}$, Hongning $\mathrm{Xie}^{2}$ and Zilian Wang ${ }^{7^{*}}$ (D)

\begin{abstract}
Background: The prevalence of both placenta previa and cesarean are on the rise. Multiple adverse outcomes are critically increased when placenta previa is subsequent to prior cesarean. The purpose of the present study is to develop a pre-surgical method for predicting adverse outcomes in pregnancy complicated with both placenta previa and prior cesarean.

Methods: Clinical data was obtained from the medical history system at the First Affiliated Hospital of Sun Yat-sen University from February 2003 to December 2016. All cases with a final diagnosis of "placenta previa/low lying placenta (ICD:044.001-105)" and "scarred uterus complicated with pregnancy (ICD: O34.200-202)" were collected and reviewed. Hysterectomy was taken as the primary outcome; and blood loss was taken as the secondary outcome.

Results: Of 219 pregnant women in the final analysis, 25 received a hysterectomy following delivery, and 48 had blood loss exceeding $1000 \mathrm{ml}$. Pre-surgical risk factors for hysterectomy are ultrasonic signs of vascular lacunae, central placenta previa, and loss of normal hypoechoic retroplacental zone. A pre-surgical predictive equation referred to as "Hysterectomy Index in Placenta Previa with Prior cesarean (HIPs)" was generated and each risk factor was weighted to create an 8-point scale. This index yielded an area under the curve of 0.972 for the prediction of hysterectomy.

Conclusions: Application of the HIPs score may provide an effective pre-surgical prediction of cesarean hysterectomy in pregnant women complicated with both placenta previa and prior cesarean.
\end{abstract}

\section{Background}

Placenta accreta is associated with multiple adverse outcomes, including massive haemorrhage, cesarean hysterectomy, and maternal mortality [1-5]. Pregnant women presenting with placenta previa and prior cesarean have a higher risk of placenta accreta [6]. In recent decades, the prevalence of placenta previa-accreta has increased [7], partly due to increasing rates of cesarean births [8].

The complications of placenta previa-accreta can be life-threatening, thus, pre-operative prediction of these adverse outcomes is of great importance $[9,10]$. Several articles have provided models to predict placental

\footnotetext{
* Correspondence: wangzil@mail.sysu.edu.cn

${ }^{\dagger}$ Bin Liu and Songqing Deng are co-first authors.

'Department of Obstetrics and Gynecology, The First Affiliated Hospital of

Sun Yat-sen University, 58 Zhongshan Road II, Guangzhou 510080, People's

Republic of China

Full list of author information is available at the end of the article
}

invasion [11-13] in cases with placenta previa and prior cesarean. However, there currently are no models to predict adverse clinical outcomes in these patients.

Therefore, the purpose of the present study is to analyze related risk factors of adverse outcomes, including blood loss and hysterectomy, in pregnancies complicated with placenta previa and prior cesarean. A pre-surgical prediction system will be generated using risk factors that are associated with hysterectomy and blood loss.

\section{Materials and methods}

The present study is a retrospective analysis of risk factors relating to hysterectomy and blood loss during surgery in suspected cases of placenta previa-accreta. Clinical data was obtained from the medical history system at the First Affiliated Hospital of Sun Yat-sen University from February 2003 to December 2016. All cases with a final diagnosis of "placenta previa/low lying 
placenta (ICD:O44.001-105)" and "scarred uterus complicated with pregnancy (ICD: O34.200-202)" were collected for primary analysis. This study was approved by the ethical committees of The First Affiliated Hospital of Sun Yat-sen University (2017-323).

Medical records were reviewed and clinical, laboratory, and ultrasonic information was collected. Clinical and laboratory information included maternal age, gestational age at delivery, time elapsed since last cesarean, number of prior cesareans, prior curettage, pregravid and prepartum BMI, neonatal birth weight, Apgar scores, and results of last hemoglobulin test prior to delivery. Ultrasonic information included four major signs related to placenta invasion (vascular lacunae, loss of normal hypoechoic retroplacental zone, retroplacental myometrial thinness, and placental thickness), as well as type of placenta previa (central, partial, marginal or low-lying) and placenta position (anterior, posterior or sidewall).

Hysterectomy was taken as the primary outcome and blood loss as the secondary outcome. The relationship between clinical, laboratory, and ultrasonic information and adverse outcomes were analyzed. Continuous and normally distributed variables were analyzed by independent sample $t$ test. Categorical variables were examined with Chi-square test. Related factors with statistical significance were further scrutinized using linear logistic regression.

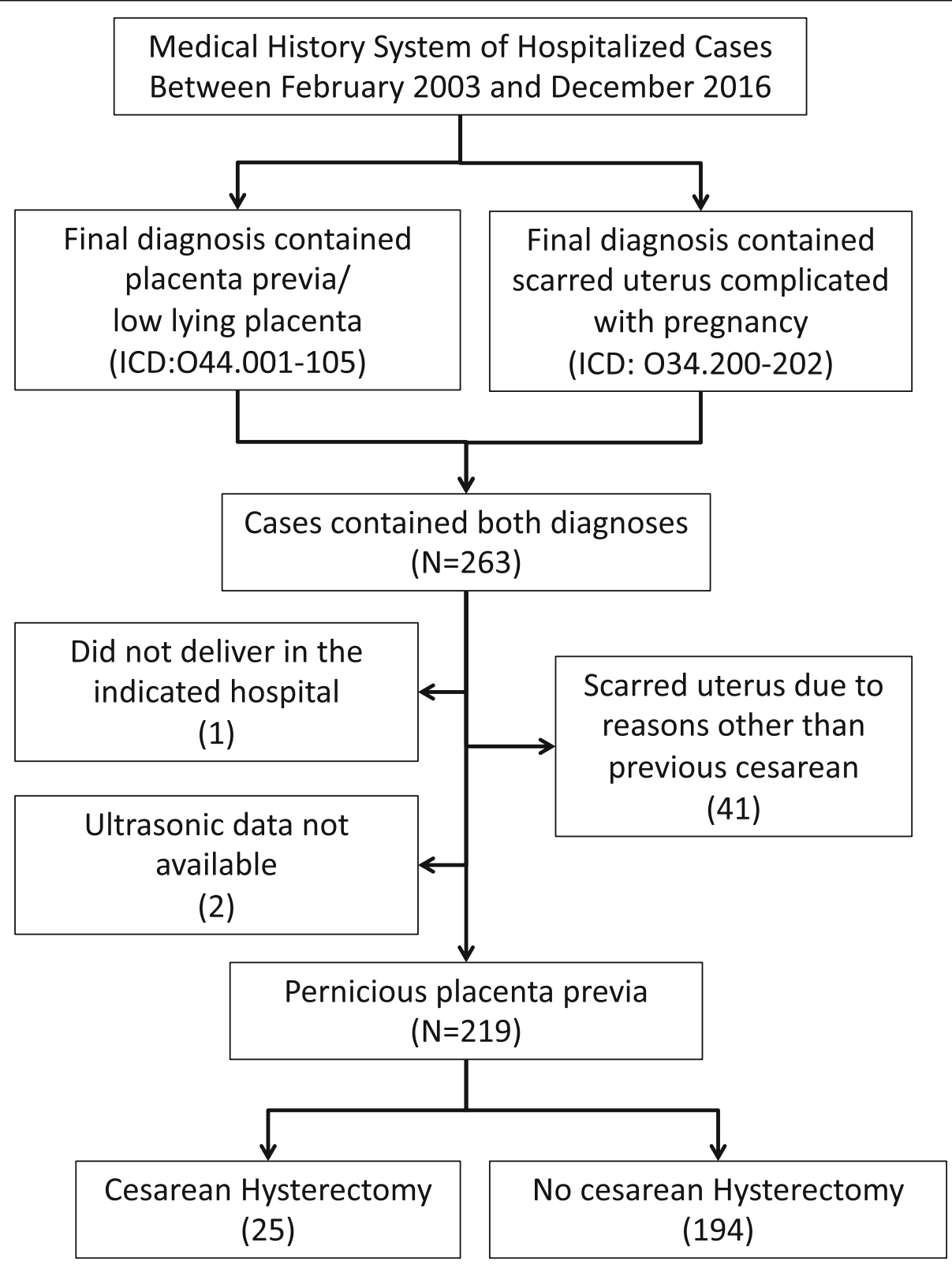

Fig. 1 Process of clinical data collection and selection 
Table 1 Basal characteristics of research population

\begin{tabular}{llll}
\hline & Hysterectomy & Control & $P$ \\
& & & value \\
\hline Number & 25 & 194 & \\
Demographic characteristics and medical history & & \\
Age (Year) & $34.08 \pm 4.42$ & $33.82 \pm 4.13$ & 0.773 \\
Gestational age (Day) & $242.44 \pm$ & $249.01 \pm$ & 0.371 \\
& 31.53 & 34.74 & \\
Time since last surgery & $55.52 \pm 29.74$ & $65.46 \pm 37.64$ & 0.205 \\
(Month) & & & \\
Gestational diabetes mellitus & $3(12)$ & $38(19.6)$ & 0.585 \\
(\%) & & & \\
Hypertensive disorder (\%) & $0(0)$ & $7(3.6)$ & 1.000 \\
Prior Cesarean >=2 times (\%) & $5(20.0)$ & $14(7.2)$ & 0.049 \\
Prior curettage (\%) & $17(68.0)$ & $90(46.4)$ & 0.055 \\
Bleeding prior to delivery (\%) & $10(40.0)$ & $57(29.4)$ & 0.356 \\
Physical and laboratory examinations & & & \\
Pregravid BMl (kg/m²) & $21.83 \pm 2.66$ & $21.91 \pm 2.85$ & 0.911 \\
Prepartum BMl (kg/m²) & $26.09 \pm 3.50$ & $26.30 \pm 2.90$ & 0.739 \\
Last Hb before delivery (g/L) & $103.04 \pm$ & $114.12 \pm$ & $<$ \\
& 10.94 & 15.08 & 0.001 \\
Neonatal information & & & \\
Birth weight (kg) & $2.45 \pm 0.62$ & $2.78 \pm 0.80$ & 0.057 \\
Apgar 1 min & $9.23 \pm 0.92$ & $9.40 \pm 1.51$ & 0.599 \\
Apgar 5 min & $9.77 \pm 0.61$ & $9.74 \pm 1.18$ & 0.899 \\
Apgar 10 min & $9.82 \pm 0.50$ & $9.80 \pm 1.14$ & 0.931 \\
\hline
\end{tabular}

$\mathrm{Hb}$ Hemoglobulin

$B M I$ Body Mass Index

By applying all significant factors related to hysterectomy, we generated an evaluation system referred to as "Hysterectomy Index in Placenta Previa with Prior cesarean (HIPs)". Each related factor was weighted to create a scale and the sensitivity and specificity were calculated for each score.

Table 2 Ultrasonic signs related to hysterectomy and blood loss in surgery

\begin{tabular}{llll}
\hline & Hysterectomy & Control & $\begin{array}{l}P \\
\text { value }\end{array}$ \\
& & & \\
\hline Number & 25 & 194 & \\
Vascular lacunae (\%) & $17(68)$ & $16(8.2)$ & $<$ \\
& & & 0.001 \\
& $22(88)$ & $9(4.6)$ & $<$ \\
$\begin{array}{l}\text { Loss of normal hypoechoic } \\
\text { retroplacental zone (\%) }\end{array}$ & & 0.001 \\
Retroplacental myometrial thinness (\%) & $4(16)$ & $5(2.6)$ & 0.011 \\
Placental thickness (\%) & $12(48)$ & $8(4.1)$ & $<$ \\
& & & 0.001 \\
Central Placenta previa (\%) & $22(88)$ & $56(28.9)$ & $<$ \\
& & & 0.001 \\
Anterior Placenta previa (\%) & $25(100)$ & $102(54.8)$ & $<$ \\
& & & 0.001 \\
\hline
\end{tabular}

Table 3 Predictors of hysterectomy in pregnant women complicated with placenta previa and prior cesarean

\begin{tabular}{llll}
\hline & $\begin{array}{l}\text { Regression } \\
\text { coefficient }\end{array}$ & OR $(95 \% \mathrm{Cl})$ & $\begin{array}{l}P \\
\text { value }\end{array}$ \\
\hline Vascular lacunae & 2.041 & $\begin{array}{l}7.701(1.445, \\
41.051)\end{array}$ & 0.017 \\
Central placenta previa & 2.436 & $\begin{array}{l}11.429(1.395, \\
\text { 93.618) }\end{array}$ & 0.023 \\
& & $32.246(5.886$, & $<$ \\
$\begin{array}{l}\text { Loss of normal hypoechoic } \\
\text { retroplacental zone }\end{array}$ & 3.473 & $176.642)$ & 0.001 \\
\hline
\end{tabular}

Input variables include: number of cesarean $>1$, prior curettage, last $\mathrm{Hb}$ before delivery, ultrasonic sign of vascular lacunae, loss of normal hypoechoic retroplacental zone, retroplacental myometrial thinness, placental thickness, central placenta previa, and anterior placenta previa

\section{Results}

Reviewing the database, 263 pregnant women met the inclusion criteria: diagnosis of both placenta previa and scarred uterus. Of all cases, 41 were excluded because their uterine scars were due to reasons other than previous cesarean (i.e. myomectomy), one was excluded because the patient did not deliver in our hospital, and two were excluded because ultrasonic data were not obtained due to emergency surgery. Of the remaining 219 cases in the final analysis, 25 received surgical hysterectomy (Fig. 1).

Basal maternal and neonatal information for hysterectomy and control cases are shown in Table 1. There were no statistically significant differences in age, gestational age, time since last surgery, prevalence of GDM or hypertensive disorder, bleeding history prior to delivery, pregravid and prepartum BMI, neonatal birthweight and Apgar scores. The hysterectomy group had higher incidence of two or more prior cesarean deliveries $(20.0 \%$ vs $7.2 \%, P=0.049)$, as well as lower hemoglobulin levels prior to delivery $(103.04 \pm 10.94 \mathrm{~g} / \mathrm{L}$ vs $114.12 \pm 15.08 \mathrm{~g} /$ $\mathrm{L}, P<0.001)$. The hysterectomy group also had a higher incidence of prior curettage but did not reach statistical significance $(68.0 \%$ vs $46.4 \%, P=0.055)$.

Since ultrasonic examination plays an important role in the prediction of surgery risks, we analyzed the characteristics of ultrasound images in detail. As shown in Table 2, four signs indicating placenta invasion including vascular lacunae, loss of normal hypoechoic retroplacental zone, retroplacental myometrial thinness, and placental thickness, were all more prevalent in cases with hysterectomy. In addition, the incidence of central placenta previa and

Table 4 Hysterectomy Index in Placenta Previa with Prior cesarean (HIPs)

\begin{tabular}{ll}
\hline & Score \\
\hline Vascular lacunae & 2 \\
Central placenta previa & 2.5 \\
Loss of normal hypoechoic retroplacental zone & 3.5 \\
\hline
\end{tabular}


anterior placenta previa were higher in patients that received hysterectomy.

In the hysterectomy group, placenta adherence was observed in all cases with gross pathological examination, and 23 of them were further confirmed by microscopic pathology. In the 2 cases without description of placenta adherence in microscopic pathologic records, the gross manifestation of placenta adherence was obvious. One potential reason for the lack of microscopic pathology manifestation of these 2 cases may be inadequate selection of tissue from the uterus for microscopic examination.

To screen for risk factors that were associated with hysterectomy in these cases, we used logistic regression to analyze each clinical, laboratory, and ultrasonic parameter that was different in each group. As shown in Table 3, linear logistic regression demonstrated that ultrasonic indication of central placenta previa, vascular lacunae, and loss of normal hypoechoic retroplacental zone were associated with hysterectomy.

Next, we generated an 8-point scale to predict the probability of hysterectomy in pernicious placenta previa cases, termed the "Hysterectomy Index in Placenta Previa with Prior cesarean (HIPs)" score (Table 4). The probability of hysterectomy for each HIPs score is shown in Table 5, with the greatest area under the receiver operating characteristic curve of HIPs at 0.972 (Fig. 2).

In the present study, there was no loss of life, therefore risk factors associated with maternal mortality was not studied. However, blood loss during surgery is an important risk factor of maternal mortality, also related to the decision of hysterectomy. Therefore, we studied risk factors that related to massive blood loss (more than $1000 \mathrm{ml}$ during surgery). We found that ultrasonic indication of loss of normal hypoechoic retroplacental zone, placenta thickness, and central placenta previa were related to blood loss during surgery (Table 6).

\section{Discussion}

The main challenge to clinical obstetricians on management of placenta previa-accreta is that more than half of the cases were not diagnosed prior to cesarean $[14,15]$.

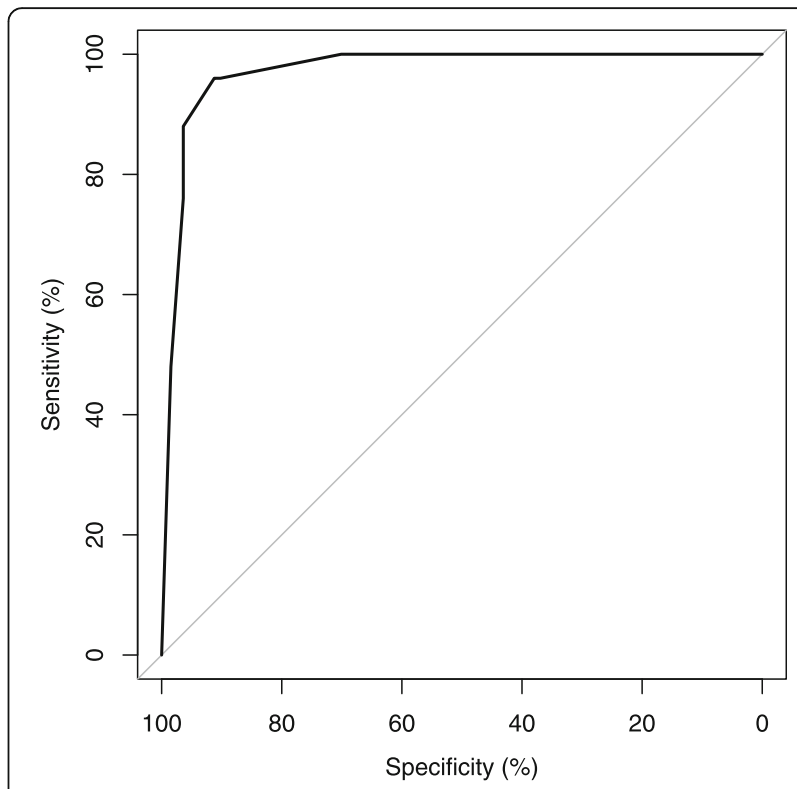

Fig. 2 Receiver operator curves of Hysterectomy Index in Placenta Previa with Prior cesarean (HIPs). The AUC of HIPs on prediction of hysterectomy in indicative cases was 0.972(95\% Cl: 0.949-0.994)

Low pre-operative diagnosis rates can lead to increased blood loss, cesarean hysterectomy, and maternal mortality. In the present study, we analyzed related factors of cesarean hysterectomy and blood loss in suspected cases of placenta previa-accreta, and developed a predictive system termed "Hysterectomy Index in Placenta Previa with Prior cesarean (HIPs)".

The application of HIPs can pre-operatively predict risk of cesarean hysterectomy in suspected cases, with a receiver operator curve of 0.972 . Three parameters, including ultrasonic image of central placenta previa, vascular lacunae, and loss of normal hypoechoic retroplacental zone, were screened to form the HIPs point system with a total score of 8 . For example, a pregnant woman with ultrasonic finding of central placenta previa and loss of normal hypoechoic retroplacental zone would receive a total score of 6 , and the predicted incidence of hysterectomy would be $54.430 \%$.

Table 5 Probability of hysterectomy, and sensitivity, specificity, positive and negative predictive values of HIPS scores

\begin{tabular}{llllllll}
\hline HIPs Score & $\mathrm{N}$ & Hysterectomy (\%) & Probability of Hysterectomy (\%) & Sensitivity (\%) & Specificity (\%) & PPV (\%) & NPV (\%) \\
\hline$>=2$ & 3 & $0(0)$ & 1.867 & 100 & 68.6 & 29.1 & 100 \\
$>=2.5$ & 40 & $1(2.5)$ & 3.093 & 100 & 70.1 & 30.0 & 100 \\
$>=3.5$ & 2 & $0(0)$ & 8.243 & 96 & 90.2 & 55.8 & 99.4 \\
$>=4.5$ & 12 & $2(16.7)$ & 20.184 & 96 & 91.2 & 58.5 & 99.4 \\
$>=5.5$ & 3 & $3(100)$ & 41.585 & 88 & 96.4 & 75.9 \\
$>=6$ & 11 & $7(63.6)$ & 54.430 & 90.445 & 46 & 96.4 & 98.4 \\
$=8$ & 15 & $12(80.0)$ & & & 98.5 & 80.1 & 96.9 \\
\hline
\end{tabular}

PPV positive predictive value

NPV negative predictive value 
Table 6 Risk factors associated with massive blood loss in pregnant women complicated with placenta previa and prior cesarean

\begin{tabular}{|c|c|c|c|c|c|}
\hline & $>=1000 \mathrm{~mL}$ & $<1000 \mathrm{~mL}$ & $P$ Value & Adjusted OR (95\%Cl) & Adjusted $P$ value \\
\hline N & 48 & 171 & & & \\
\hline Age (Years) & $34.10 \pm 4.00$ & $33.78 \pm 4.20$ & 0.638 & & \\
\hline Gestational age (Days) & $249.8 \pm 22.9$ & $247.8 \pm 36.9$ & 0.726 & & \\
\hline Time since last surgery (Months) & $58.1 \pm 33.3$ & $66.1 \pm 37.8$ & 0.188 & & \\
\hline Gestational diabetes mellitus (\%) & $11(22.9)$ & $30(17.5)$ & 0.407 & & \\
\hline Hypertensive disorder (\%) & 0 & $7(4.1)$ & 0.352 & & \\
\hline Prior Cesarean $>=2$ times (\%) & $10(20.8)$ & $9(5.3)$ & 0.002 & & \\
\hline Prior curettage (\%) & $29(60.4)$ & 78(45.6) & 0.075 & & \\
\hline Bleeding prior to delivery (\%) & 19(39.6) & $48(28.1)$ & 0.156 & & \\
\hline Pregravid BMI $\left(\mathrm{kg} / \mathrm{m}^{2}\right)$ & $22.20 \pm 2.70$ & $21.80 \pm 2.86$ & 0.403 & & \\
\hline Prepartum BMI $\left(\mathrm{kg} / \mathrm{m}^{2}\right)$ & $26.42 \pm 3.13$ & $26.24 \pm 2.92$ & 0.711 & & \\
\hline Last $\mathrm{Hb}$ before delivery $(\mathrm{g} / \mathrm{L}$ ) & $106.60 \pm 13.17$ & $114.61 \pm 15.13$ & 0.001 & & \\
\hline Vascular lacunae & $24(50.0)$ & $9(5.3)$ & $<0.001$ & & \\
\hline Loss of normal hypoechoic retroplacental zone & $29(60.4)$ & $2(1.2)$ & $<0.001$ & $115.933(11.913,1128.228)$ & $<0.001$ \\
\hline Retroplacental myometrial thinness & $6(12.5)$ & $3(1.8)$ & 0.004 & & \\
\hline Placental thickness & 19(39.6) & $1(0.6)$ & $<0.001$ & $77.542(4.394,1368.455)$ & 0.003 \\
\hline Central Placenta previa & $36(75.0)$ & $42(24.6)$ & $<0.001$ & $3.563(1.287,9.861)$ & $<0.001$ \\
\hline Anterior Placenta position & 39(81.3) & $88(54.0)$ & 0.001 & & \\
\hline
\end{tabular}

Input parameters in logistic regression were: Prior Cesarean > = 2 times, Last Hb before delivery, vascular lacunae, loss of normal hypoechoic retroplacental zone, placental thickness, central placenta previa, anterior placenta position

By using the HIPs score, cases with high risk of hysterectomy may be identified prior to operation, allowing additional time for full pre-surgical preparation, including ureteral stent insertion [16], verifying sufficient blood bank supplies, and arrangements for multidisciplinary therapy. In addition, patients with high risk of hysterectomy may be informed prior to surgery, which will reduce the risk of medical dispute.

In the HIPs system, ultrasonic evaluation of placental invasion plays a very important role. Ultrasonic signs indicating placental invasion, such as vascular lacunae and loss of normal hypoechoic retroplacental zone [12, 17], were included in the HIPs scoring system. This finding is consistent with previous reports on risk of placenta accreta [12]. In addition, loss of normal hypoechoic retroplacental zone and placenta thickness, other placental invasion signs, were related to massive blood loss in the present study. In a study by Yosuke Baba et al, ultrasound signs of lacunae was associated with allogeneic blood transfusion in cesarean section for placenta previa [18]. Jung-Won Kim et al found that ultrasonic signs of invasion was associated with massive transfusion in placenta previa cases [19]. These findings indicate that placenta accreta is the major cause of adverse outcomes in these cases.

There are several limitations of the present study. First, this is a retrospective study, so the predictive power of HIPs should be confirmed by a prospective study. Second, placental invasion was not confirmed in all cases, since only 25 patients received hysterectomy. A previous study [12] only included cases with histologic confirmation, so the information of those suspected patients was lost. In the present study, we focused on hysterectomy, a confirmed clinical outcome, rather than placental invasion in the whole cohort.

Despite these limitations, the present study developed a predictive system of cesarean hysterectomy in suspected cases of placenta previa-accreta, based on a full review of risk factors in a cohort spanning 10 years. To the best of our knowledge, this is the first analysis focused on adverse clinical results, including cesarean hysterectomy and massive blood loss in pregnant women complicated with both placenta previa and prior cesarean.

\section{Conclusion}

In conclusion, the HIPs index may help clinical doctors identify high risk cases, so that more precise counseling and full preparation for delivery can be made to improve clinical outcomes. The predictive value of HIPs may be examined in subsequent prospective observations.

\section{Acknowledgements}

We appreciate faculty and staff in the department of OBGYN, The First Affiliated Hospital, Sun Yat-sen University for their support of this study.

\section{Authors' contributions}

$\mathrm{BL}$ and $\mathrm{SD}$ designed the study. $\mathrm{ML}, J J C$ and $L S$ contributed to the acquisition of data, and analyzed data with JC, JY and BL. YC and JZ provide statistical 
analysis of the data. BL and SD drafted the manuscript. HX and ZW critically revised the draft manuscript. All authors read and approved the final manuscript.

\section{Funding}

The present study is funded by the National Natural Science Foundation of China (No. 81771602, to Bin Liu), Natural Science Foundation of Guangdong Province (No. 2017A030313826, to Bin Liu), Sun Yat-Sen University Clinical Research 5010 Program (No.2016014, to Bin Liu), Fundamental Research Funds for the Central Universities (No. 16ykpy19, to Bin Liu), and Science and Technology Planning Project of Guangdong Province (No. 2017A020214014, to Zilian Wang). The funding bodies had no role in the design of study, collection, analysis and interpretation of data, or in writing of the manuscript.

\section{Availability of data and materials}

Data of the present research is available by contacting the corresponding author on reasonable request.

\section{Ethics approval and consent to participate}

This study was approved by the ethical committee of The First Affiliated Hospital of Sun Yat-sen University (2017-323). The ethics committee waived the need for informed consent due to the retrospective study design.

\section{Consent for publication}

Not applicable.

\section{Competing interests}

The authors declare that they have no conflict of interest.

\section{Author details}

'Department of Obstetrics and Gynecology, The First Affiliated Hospital of Sun Yat-sen University, 58 Zhongshan Road II, Guangzhou 510080, People's Republic of China. ${ }^{2}$ Department of Ultrasound, The First Affiliated Hospital of Sun Yat-sen University, Guangzhou 510080, People's Republic of China. ${ }^{3}$ Department of Medical Statistics and Epidemiology, School of Public Health, Sun Yat-sen University, Guangzhou 510080, People's Republic of China.

Received: 1 June 2018 Accepted: 4 February 2020

Published online: 07 February 2020

\section{References}

1. Vahanian SA, Lavery JA, Ananth CV, et al. Placental implantation abnormalities and risk of preterm delivery: a systematic review and metaanalysis. Am J Obstet Gynecol. 2015;213(4 Suppl):S78-90.

2. Mercer B, Berghella V, Foley M, et al. Publications Committee, Society for Maternal-Fetal Medicine. Belfort: Collaborators in the Publications Committee, Society for Maternal-Fetal Medicine. https://doi.org/10.1016/j. ajog.2010.09.013.

3. Miller DA, Chollet JA, Goodwin TM. Clinical risk factors for placenta previaplacenta accreta. Am J Obstet Gynecol. 1997;177(1):210-4.

4. Silver RM, Landon MB, Rouse DJ, et al. Maternal morbidity associated with multiple repeat cesarean deliveries. Obstet Gynecol. 2006;107(6):1226-32.

5. Warshak CR, Ramos GA, Eskander R, et al. Effect of predelivery diagnosis in 99 consecutive cases of placenta accreta. Obstet Gynecol. 2010;115(1):65-9.

6. Usta IM, Hobeika EM, Musa AA, et al. Placenta previa-accreta: risk factors and complications. Am J Obstet Gynecol. 2005;193(3 Pt 2):1045-9.

7. Floode KM, Said S, Geary M, et al. Changing trends in peripartum hysterectomy over the last 4 decades. Am J Obstet Gynecol. 2009;200(6):632 e1-6.

8. Cheng PJ, Duan T. China's new two-child policy: maternity care in the new multiparous era. BJOG. 2016;123(Suppl 3):7-9.

9. Chantraine F, Braun T, Gonser M, et al. Prenatal diagnosis of abnormally invasive placenta reduces maternal peripartum hemorrhage and morbidity. Acta Obstet Gynecol Scand. 2013;92(4):439-44

10. Weiniger $C F$, Einav $S$, Deutsch $L$, et al. Outcomes of prospectively-collected consecutive cases of antenatal-suspected placenta accreta. Int J Obstet Anesth. 2013;22(4):273-9.

11. Hall T, Wax JR, Lucas FL, et al. Prenatal sonographic diagnosis of placenta accreta-impact on maternal and neonatal outcomes. J Clin Ultrasound. 2014;42(8):449-55.
12. Rac MW, Dashe JS, Wells CE, et al. Ultrasound predictors of placental invasion: the Placenta Accreta Index. Am J Obstet Gynecol. 2015;212(3):343 e1-7.

13. Tanimura $\mathrm{K}$, Morizane $\mathrm{M}$, Deguchi $\mathrm{M}$, et al. A novel scoring system for predicting adherent placenta in women with placenta Previa. Placenta. 2018;64:27-33

14. Fitzpatrick KE, Sellers S, Spark P, et al. The management and outcomes of placenta accreta, increta, and percreta in the UK: a population-based descriptive study. BJOG. 2014;121(1):62-70 discussion -1.

15. Thurn $L$, Lindqvist $P G$, Jakobsson $M$, et al. Abnormally invasive placentaprevalence, risk factors and antenatal suspicion: results from a large population-based pregnancy cohort study in the Nordic countries. BJOG. 2016;123(8):1348-55

16. Matsubara S, Kuwata T, Usui R, et al. Important surgical measures and techniques at cesarean hysterectomy for placenta previa accreta. Acta Obstet Gynecol Scand. 2013;92(4):372-7.

17. Bowman ZS, Eller AG, Kennedy AM, et al. Accuracy of ultrasound for the prediction of placenta accreta. Am J Obstet Gynecol. 2014;211(2):177 e1-7.

18. Baba Y, Ohkuchi A, Usui R, et al. Calculating probability of requiring allogeneic blood transfusion using three preoperative risk factors on cesarean section for placenta previa. Arch Gynecol Obstet. 2015;291(2):2815.

19. Kim JW, Lee YK, Chin JH, et al. Development of a scoring system to predict massive postpartum transfusion in placenta previa totalis. J Anesth. 2017; 31(4):593-600

\section{Publisher's Note}

Springer Nature remains neutral with regard to jurisdictional claims in published maps and institutional affiliations.
Ready to submit your research? Choose BMC and benefit from:

- fast, convenient online submission

- thorough peer review by experienced researchers in your field

- rapid publication on acceptance

- support for research data, including large and complex data types

- gold Open Access which fosters wider collaboration and increased citations

- maximum visibility for your research: over $100 \mathrm{M}$ website views per year

At BMC, research is always in progress.

Learn more biomedcentral.com/submissions 